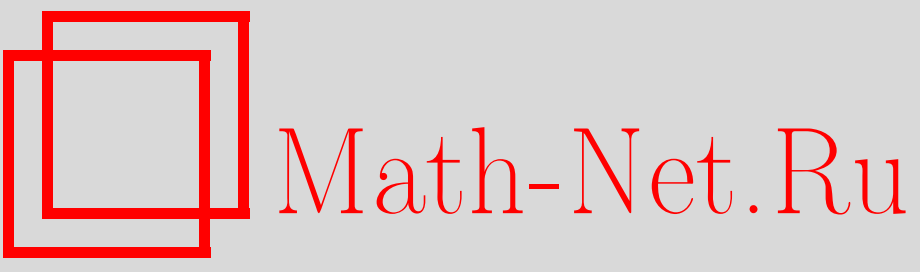

А. С. Джумадильдаев, К. М. Туленбаев, Бикоммутативные алгебры, УМH, 2003, том 58, выпуск 6, 149-150

DOI: https://doi.org/10.4213/rm681

Использование Общероссийского математического портала Math-Net.Ru подразумевает, что вы прочитали и согласны с пользовательским соглашением

http://www.mathnet.ru/rus/agreement

Параметры загрузки:

IP : 52.6 .47 .48

26 апреля 2023 г., $17: 49: 14$ 


\title{
БИКОММУТАТИВНЫЕ АЛГЕБРЫ
}

\author{
А. С. ДЖУМАДИЛЬДАЕВ, К. М. ТУЛЕНБАЕВ
}

Алгебра над полем $K$ назьвается левокоммутативной, если выполнено тождество $a \circ(b \circ c)=$ $b \circ(a \circ c)$. Аналогично правокоммутативная алгебра определяется тождеством $(a \circ b) \circ c=(a \circ c) \circ b$. Право- и левокоммутативные алгебры называются бикоммутативными.

Алгебра матриц порядка $n$ удовлетворяет стандартному тождеству степени $2 n$ [1]. Такие тождества можно рассматривать как обобщения условия коммутативности. Интерес к односторонне коммутативным алгебрам мотивирован потребностями теории скобок Пуассона и интегрируемых систем. Такие алгебры возникают, например, при рассмотрении уравнений гидродинамического типа [2].

Имеется бесконечно много неизоморфных простых односторонне коммутативных алгебр. Например, алгебра с базисом $\left\{e_{1}, \ldots, e_{n}\right\}$ и умножением $e_{i}$ ○ $e_{j}=\lambda_{i j} e_{j}$, где $\left(\lambda_{i j}\right)$ - матрица с различными столбцами, дает пример простых левокоммутативных алгебр [3].

Пусть $A^{-}$и $A^{+}$- алгебры, полученные из $A$, с новыми умножением $[a, b]=a \circ b-b \circ a$ и $\{a, b\}=a \circ b+b \circ a$, соответственно.

Теорема 1. Пусть $A$ - бикоммутативная алгебра. Тогда $A^{2}$ коммутативна и ассочиативна. В частности, всякая простая конечномерная алгебра одномерна. Кроме того, в А имеют место тождества

$$
a \circ[b, c]+b \circ[c, a]+c \circ[a, b]=0, \quad[a, b] \circ c+[b, c] \circ a+[c, a] \circ b=0 .
$$

В частности, $A^{-}$является алгеброй Ли. В алгебре $A^{+}$имеют место тождества

$$
\{a, b\}=\{b, a\}, \quad\{\{a, b\},\{c, d\}\}-\{\{a, d\},\{c, b\}\}=-\left\{(a, b, c)^{+}, d\right\}+\left\{(a, d, c)^{+}, b\right\},
$$

əде $(a, b, c)^{+}=\{a,\{b, c\}\}-\{\{a, b\}, c\}-$ ассоциатор в алгебре $A^{+}$.

ЗАмечание. Если $A$ - алгебра Новикова, то $A^{+}$удовлетворяют тождествам

$$
\{a, b\}=\{b, a\}, \quad\{\{a, b\},\{c, d\}\}-\{\{a, d\},\{c, b\}\}=\left\{(a, b, c)^{+}, d\right\}-\left\{(a, d, c)^{+}, b\right\} .
$$

Приведем классификацию бикоммутативных алгебр $A$ размерности $\leqslant 3$ с условием $A^{2}=A$. Всякая двумерная бикоммутативная алгебра изоморфна алгебре с базисом $\{a, b\}$ и с умножениями

$$
a \circ a=a, \quad a \circ b=b \circ a=\varphi a, \quad b \circ b=b+\left(\varphi^{2}-\varphi\right) a .
$$

Для трехмерных алгебр имеется два класса неизоморфных алгебр. Пусть $a, b, c-$ базисные элементы. Умножения в первом классе определяются так:

$$
\begin{gathered}
a \circ a=a+\left(\lambda^{2}-\lambda\right) c, \quad a \circ c=c \circ a=\lambda c, \\
b \circ c=c \circ b=\mu c, \quad a \circ b=b \circ a=\varphi a+\lambda(\mu-\varphi) c, \\
b \circ b=b+\varphi(\varphi-1) a+(\mu-\varphi)(\mu+\varphi-1) \lambda c, \quad c \circ c=c .
\end{gathered}
$$

Умножения во втором классе алгебр определяются так:

$$
\begin{gathered}
a \circ a=a+\lambda(2 \varphi-1) /\left(\varphi^{2}-\varphi\right) c, \quad a \circ c=c \circ a=c, \\
b \circ c=c \circ b=\varphi c, \quad a \circ b=b \circ a=\varphi a+\lambda c, \\
b \circ b=b+\varphi(\varphi-1) a, \quad c \circ c=0 .
\end{gathered}
$$

Здесь $\varphi, \mu, \lambda \in K$. 
Теорема 2. Пусть $F(q)$ - свободная бикоммутативная алгебра с q образующими и $F_{n}(q)$ - ее однородная компонента, порожденная әлементами степени $n$. Тогда

$$
\begin{aligned}
\operatorname{dim} F_{n}(q)= & \sum_{k=1}^{[n / 2]-2}\left(\begin{array}{c}
q+k \\
q-1
\end{array}\right)\left(\begin{array}{c}
q+n-k-2 \\
q-1
\end{array}\right) \\
& +\sum_{k=1}^{[n / 2]-1}\left(\begin{array}{c}
q+k \\
q-1
\end{array}\right)\left(\begin{array}{c}
q+n-k-2 \\
q-1
\end{array}\right)+2 q\left(\begin{array}{c}
n+q-2 \\
q-1
\end{array}\right) .
\end{aligned}
$$

СлеДСтвиЕ. Для однопорожденной алгебры $\operatorname{dim} F_{n}(1)=n$ и ряд Гильберта задается $\operatorname{ma\kappa } H_{t}=1-t+t^{3} /(1-t)^{2}$.

Свободньй базис алгебры $F(q)$ можно описать в терминах корневых деревьев [4] следующих двух типов:
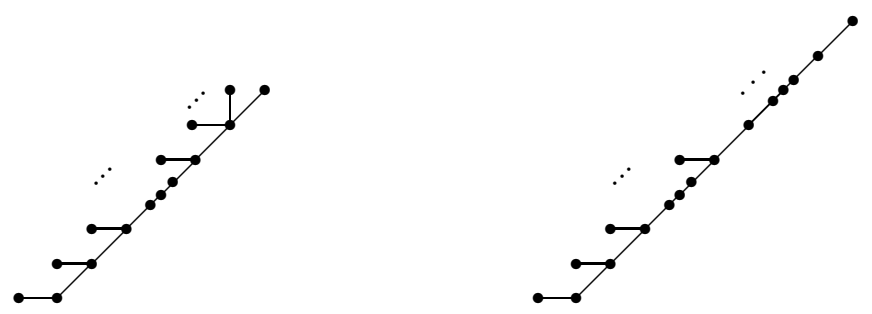

Вершины таких деревьев нумеруются образующими алгебры $F(q)$. Условия бикоммутативности накладьвают различные условия на вершины деревьев. Например, разрешается переставлять ветки и вершины вдоль ствола. Классы изоморфизмов таких деревьев дают базис алгебры $F(q)$.

Авторы благодарны программе ИНТАС за поддержку.

\section{СПИСОК ЛИТЕРАТУРЫ}

[1] S. A. Amitsur, J. Levitzki // Proc. Amer. Math. Soc. 1950. V. 1. P. 449-463. [2] А. А. Балинский, С. П. Новиков // Докл. АН СССР. 1985. Т. 283. № 5. С. 1036-1039. [3] И.П. Шестаков, Е.Н. Кузьмин // Итоги науки и техники. Соврем. пробл. матем. Фундам. напр. Т. 57. М.: ВИНИТИ, 1990. С. 179-266. [4] A. Cayley. On the theory of analitical forms called trees // Phil. Mag. 1857. V. 13. P. 19-30; Math. Papers, Cambridge. 1891. V. 3. P. 242-246.

Институт математики НАН Республики Казахстан, Алматы

Принято редколлегией E-mail: askar@math.kz 